\title{
Clinical spectrum associated with positive ANCA titres in 94 consecutive patients: is there a relation with PR-3 negative c-ANCA and hypergammaglobulinaemia?
}

\author{
D Blockmans, E Stevens, G Mariën, H Bobbaers
}

\begin{abstract}
Objective-To calculate the positive predictive value (ppv) of cytoplasmic antineutrophil cytoplasmic antibodies (cANCAs) and anti-proteinase 3 (PR 3) antibodies for Wegener's granulomatosis (WG) and to evaluate their association with other diseases.
\end{abstract}

Methods-The clinical files of all 94 patients who had a positive $c-$ or perinuclear (p)-ANCA test, or both, in the laboratory of the University Hospital, Leuven between April 1995 and March 1996 and who attended the Internal Medicine Department of the hospital were retrospectively studied.

Results-Of the 94 patients with ANCAs (fluorescence titre $\geqslant 1 / 40$ ), 57 were c-ANCA positive and $45 \mathrm{p}$-ANCA positive (eight were simultaneously $c-$ and p-ANCA positive). Of the $57 \mathrm{c}$-ANCA positive patients, 23 had WG. The ppv for WG thus was $40 \%$. This value did not increase by defining a higher threshold for a positive ANCA. There was not a good relation between ANCA titres and disease activity in the WG patients, nor was there a relation between anti-PR 3 antibody levels and WG disease activity. The ppv of anti-PR 3 antibodies for WG however was very high $(85 \%)$. There was a positive correlation between the level of (hyper) gammaglobulinaemia and c-ANCA titres in those patients with final diagnoses not known to be associated with c-ANCA. Forty five patients had positive p-ANCAs. The largest group were those with inflammatory bowel disease $(n=20$, of whom the majority had colitis ulcerosa or primary sclerosing cholangitis, or both); the great majority of these patients had no anti-myeloperoxidase antibodies. Vasculitis was present in eight patients, of whom two had WG (both were also c-ANCA positive).

Conclusion-There is a low ppv of c-ANCAs for WG, caused by a high percentage of PR 3 negative, positive c-ANCA determinations, possibly related to hypergammaglobulinaemia. Anti-PR 3 antibodies have a high ppv for WG. However, neither c-ANCA titre, nor the level of anti-PR 3 antibodies correlated with the activity of the disease.

(Ann Rheum Dis 1998;57:141-145)
In 1985, van der Woude et al ${ }^{1}$ first found the so called antineutrophil cytoplasmic antibodies (ANCA) - which were described three years earlier by Davies et al - to be connected with Wegener's granulomatosis (WG). They proposed that ANCAs were useful for diagnosis and for follow up of disease activity in patients with this form of systemic vasculitis. Later, cytoplasmic staining ANCAs (c-ANCAs, which were sensitive and specific for WG) were differentiated from perinuclear staining ANCAs (p-ANCAs), found in other vasculitides such as microscopic polyangiitis. ${ }^{3}$ Using ELISA techniques, the responsible antigen for c-ANCA staining was proved to be proteinase-3 (PR 3), ${ }^{4}$ while most p-ANCAs were directed against the myeloperoxidase (MPO) enzyme. ${ }^{5}$ In more recent years, other diseases without vasculitis were reported to be associated with ANCAs, for example, ulcerative colitis, primary biliary cirrhosis, rheumatoid arthritis, human immunodeficiency virus infection or Sjögren's syndrome. ${ }^{67}$ Hence, it has become less clear what diagnostic value should be attached to a positive ANCA determination in clinical practice.

We therefore calculated in a retrospective way the positive predictive value (ppv) of c-ANCA and of anti-PR 3 antibodies for WG and analysed the association of c- and p-ANCA with other diseases.

\section{Methods}

The clinical files of all 94 patients who had a positive c- or p-ANCA test, or both, in our laboratory during a one year period (between April 1995 and March 1996) and who attended the Internal Medicine Department of our hospital were retrospectively studied.

Vasculitis was bioptically confirmed in 20 of 23 WG patients. A total of 14 renal biopsies, 12 bronchoscopic biopsies, 10 nasal biopsies, five skin biopsies, and one open lung biopsy were performed. Among the 20 patients with histologically confirmed vasculitis, two patients fulfilled all four ACR criteria for WG, ${ }^{8} 11$ patients fulfilled three ACR criteria, six patients fulfilled two ACR criteria, and only one patient fulfilled only one ACR criterium. Of the three patients without histological proof of vasculitis, one patient fulfilled three ACR criteria and two patients fulfilled two criteria.

A WG patient was considered to be in remission (or to have inactive disease) when there were no clinical or biochemical signs after three months of ongoing inflammation (normal 
Table 1 Overview of the 94 ANCA positive patients with anti-PR 3 and anti-MPO results (total number of determinations $=44$ )

\begin{tabular}{|c|c|c|c|c|}
\hline & Patients (n) & & $\begin{array}{l}\text { Number of anti-PR } 3 \\
\text { positive (\%) }\end{array}$ & $\begin{array}{l}\text { Number of anti-MPO } \\
\text { positive (\%) }\end{array}$ \\
\hline \multirow[t]{4}{*}{ Pure c-ANCA } & 49 & $21 \mathrm{WG}$ & $11 / 11(100)$ & $0 / 11(0)$ \\
\hline & & 9 non-WG vasculitis & $1 / 1$ & $0 / 1$ \\
\hline & & $\begin{array}{l}2 \text { inflammatory bowel disease } \\
(1+\text { non-WG vasculitis })\end{array}$ & $0 / 0$ & $0 / 0$ \\
\hline & & 18 miscellaneous & $\begin{array}{l}1 / 6(17) \\
\text { (borderline) }\end{array}$ & $0 / 6(0)$ \\
\hline \multirow[t]{3}{*}{ Pure p-ANCA } & 37 & 18 inflammatory bowel disease & $0 / 9(0)$ & $1 / 9(11)$ \\
\hline & & 6 non-WG vasculitis & $0 / 6(0)$ & $3 / 6(50)$ \\
\hline & & 13 miscellaneous & $0 / 8(0)$ & $0 / 8(0)$ \\
\hline \multirow[t]{3}{*}{$\mathrm{c}-$ and $\mathrm{p}-\mathrm{ANCA}$} & 8 & $2 \mathrm{WG}$ & $0 / 1$ & $1 / 1$ \\
\hline & & 2 inflammatory bowel disease & $0 / 1$ & $0 / 1$ \\
\hline & & 4 miscellaneous & $0 / 1$ & $1 / 1$ \\
\hline
\end{tabular}

sedimentation rate and $\mathrm{C}$ reactive protein) and when the urinary sediment had returned to normal. ANCA titres were not taken into account to determine if a patient had active disease or not.

ANCA tests were performed by indirect immunofluorescence using commercially available slides (INOVA Diagnostics, San Diego, CA). Ethanol fixed neutrophils were exposed to patient serum diluted 1:40 for screening. A positive c-ANCA serum, a positive p-ANCA serum, and a negative control were included in every run. A specific anti-IgG fluorescent conjugate was used. Any positive samples were titrated in doubling dilutions until end point. When a p-ANCA pattern was seen (peripheral or diffuse nuclear staining), serum was tested for the presence of antinuclear antibodies (ANA) on Hep-2 cells (Immunoconcepts, Sacramento, CA). All positive ANA serum samples were further tested in a dilution of $1 / 40$ on formalin fixed neutrophils (INOVA Diagnostics, San Diego, CA). Those samples that gave a cytoplasmic staining pattern on formalin fixed neutrophils, were reported to be true p-ANCA samples despite the simultaneous presence of an ANA.

Results of c- and p-ANCA were reported as negative or positive $1 / 40$ to $1 / 10240$.

ELISA tests for anti-PR 3 and anti-MPO were purchased from INOVA Diagnostics (San Diego, CA). A specific enzyme labelled antiIgG conjugate was used. ELISA tests were carried out from December 1995, but only on serum samples positive in the immunofluorescence test and according the manufacturer's instructions $(\mathrm{n}=44)$. The results were expressed in arbitrary units per litre (AU/l). Samples were considered to be positive for anti-PR 3 when values were greater than $20 \mathrm{AU} / 1$ and for anti-MPO when values were greater than $5 \mathrm{AU} / 1$.

Total protein concentration was determined using Boehringer-Mannheim reagent kits and application on a Hitachi-747 (BoehringerMannheim, GNBH, Mannheim, Germany). Gammaglobulins were measured using cepharose-cellulose-polyacetate electrophoresis strips for Microsome System (Gelman Sciences, Ann Arbor, MI, USA). The gels were equilibrated with di-ethyl-barbital buffer $\mathrm{pH}$ 8.6.

Serum immune complexes were measured by $\mathrm{C} 1 \mathrm{q}$ and monoclonal rheumatoid factor binding solid phase radioimmunoassays.
STATISTICS

Data are given as mean (SEM). Correlations were done with the Spearman rank correlation. A $p$ value of $<0.05$ was considered significant.

\section{Results}

Of the 94 patients with positive $(\geqslant 1 / 40)$ ANCA determinations, 49 patients were only c-ANCA positive, 37 patients were only p-ANCA positive, and eight patients had both c-and p-ANCAs (table 1). Hence, a total of 57 patients were c-ANCA positive and a total of 45 patients were p-ANCA positive.

\section{PATIENTS WITH POSITIVE C-ANCA}

DETERMINATIONS

Twenty three of these 57 patients suffered from WG (21 were only c-ANCA positive, two patients had c- and p-ANCAs), which represents a ppv of a positive c-ANCA determination for WG of $40 \%$. Of these 23 patients, 12 were in remission after treatment and 11 had active disease.

The ppv of c-ANCAs did not increase when taking a higher titre as cut off point: if we consider only c-ANCAs at $1 / 80$ or higher as indicative for WG, the predictive value was $44 \%$, at $\geqslant 1 / 160, \geqslant 1 / 320$, and $\geqslant 1 / 640$, it was $45 \%, 46 \%$, and $38 \%$ respectively. The highest c-ANCA titre observed $(1 / 5120)$ was found in a non-WG patient.

Follow up determinations were carried out in 10 patients, three of them showing active disease. There was a considerable variation in ANCA titres, both in patients with still active disease and in patients who went into remission. In WG patients in remission, we found ANCA titres fluctuating from negative to $1 / 320$ without corresponding change in disease activity. Other patients in stable remission continued to have high ANCA titres throughout follow up (titres from $1 / 640$ to $1 / 2560$ ).

Nine other patients with positive c-ANCA determinations had a form of systemic vasculitis different from WG: one patient had renal limited vasculitis, one patient had Behçet's disease, three patients suffered from classic periarteritis nodosa, and in four patients vasculitis could not be further classified.

Combining all patients with a systemic vasculitic disorder (23 WG patients and nine non-WG vasculitis patients), our ppv of a positive c-ANCA determination for systemic vasculitis reaches $56 \%$. 


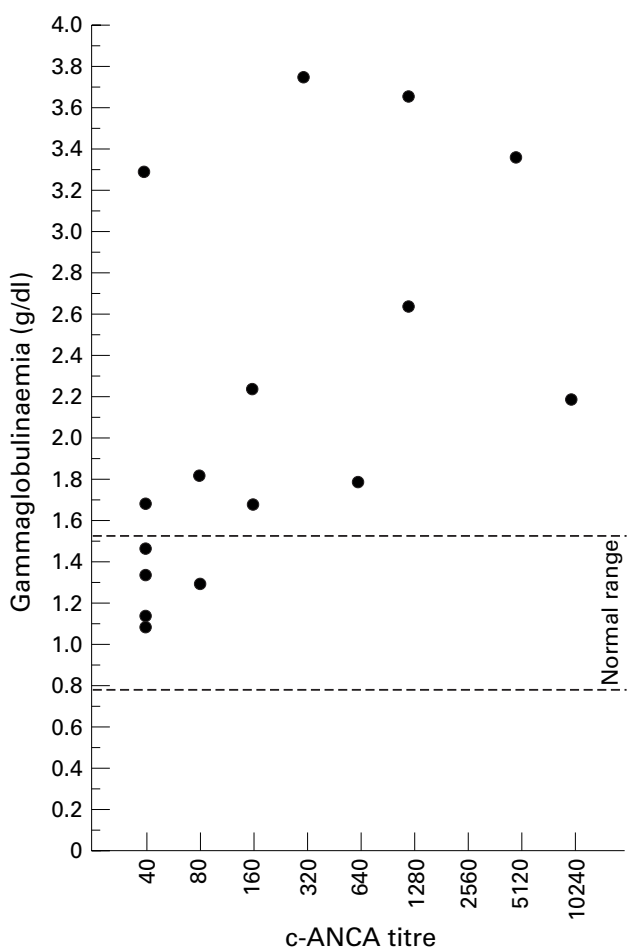

Figure 1 Gammaglobulinaemia compared with $c-A N C A$ titres in 16 patients without systemic vasculitis. Spearman rank correlation for these data gives a p value $<0.02$.

Four patients with positive c-ANCA determinations (two pure c-ANCA, two combination of c- and p-ANCA, see table 1) had colitis ulcerosa; in three patients this was associated with primary sclerosing cholangitis and in one with Behçet's disease (see above).

In the 22 remaining patients (18 patients with pure c-ANCAs, four patients with c- and p-ANCAs), no obvious reason could be found for their positive c-ANCA result. The following diagnoses were made in these patients: idiopathic lung fibrosis, bronchiectasias $(\mathrm{n}=3)$, pulmonary emphysema $(n=2)$, interstitial lung disease caused by rheumatoid arthritis, haemoptysis caused by an abnormal bronchial artery, polymyalgia rheumatica, idiopathic thrombocytopenic purpura, sarcoidosis, chronic rhinitis, IgA nephritis, urosepsis, hypersensitivity vasculitis, chronic renal failure, cirrhosis caused by hepatitis C, Sjögren's syndrome in combination with myelodysplasia, lupus nephritis, and previous hepatitis B infection $(\mathrm{n}=1)$. In 16 of these 22 patients, gammaglobulinaemia was determined and for 11 of them, the value was above the normal range (fig 1). There was a positive Spearman rank correlation $\left(r_{\mathrm{s}}=0.77, t=4.56, \mathrm{p}<0.02\right)$ between the c-ANCA titres of these patients and their gammaglobulinaemia. In WG patients, there was no positive correlation between ANCA titres and (hyper)gammaglobulinaemia. $\gamma$ Globulin concentrations in WG patients were on the average rather low (mean (SEM) $\gamma$ globulin concentration in $19 \mathrm{WG}$ patients: $0.93(0.09) \mathrm{g} / \mathrm{dl})$, probably because of immunosuppressive treatment.
PATIENTS WITH POSITIVE P-ANCA DETERMINATIONS

P-ANCAs (titre $\geqslant 1 / 40$ ) were found in 45 patients: 37 patients had pure p-ANCAs, eight patients had c- and p-ANCAs.

Twenty patients suffered from an inflammatory bowel disease or primary sclerosing cholangitis, or both: 10 patients had isolated colitis ulcerosa, seven patients had a combination of colitis ulcerosa and primary sclerosing cholangitis (of whom two were also c-ANCA positive), two patients suffered from Crohn's disease and in one patient, there was an isolated primary sclerosing cholangitis. The $\mathrm{ppv}$ of p-ANCA was $44 \%$ for the group of inflammatory bowel disease patients.

Systemic vasculitis was found in eight patients (six patients with pure p-ANCAs, two patients with c- and p-ANCAs): in two patients a diagnosis of microscopic polyangiitis was made, two patients had WG (both were also c-ANCA positive), one patient had renal limited vasculitis, one had arteritis temporalis, and in two patients vasculitis was secondary to a connective tissue disease (scleroderma and dermatomyositis respectively). Thus, the ppv of p-ANCA was $18 \%$ for this group of systemic vasculitis patients.

The 17 remaining patients with p-ANCAs (13 pure $\mathrm{p}$-ANCAs, four combination of $\mathrm{c}$ - and p-ANCAs) suffered from a wide spectrum of diseases: systemic lupus erythematosus, pneumonia $(n=2)$, emphysema, rheumatoid arthritis, Hashimoto thyroiditis, tuberculosis, bronchiectasias, extrinsic allergic alveolitis, autoimmune haemolytic anaemia, idiopathic lung fibrosis, chronic obstructive lung disease, ankylosing spondylitis, hepatitis $B$, spinal canal stenosis, unexplained isolated arthralgias $(\mathrm{n}=$ 1). The $\gamma$ globulin value was known in 13 of these 17 patients. Seven patients had normal values of $\gamma$ globulin, there was a borderline increase in one patient, and in five other patients there was hypergammaglobulinaemia. There was no correlation between p-ANCA titres and $\gamma$ globulin values.

\section{ANTI-PR 3 AND ANTI-MPO ANTIBODY} DETERMINATION

Anti-PR 3 antibodies were only found in c-ANCA positive serum samples, never in pure p-ANCA serum samples. The opposite was true for anti-MPO antibodies.

Figure 2 shows anti-PR 3 values obtained in four WG patients with active disease, seven WG patients in remission, and in seven patients with conditions other than WG (all pure c-ANCA serum samples). As figure 2 shows, there is no difference in anti-PR 3 concentrations between WG patients with still active disease and patients in remission. Patients with conditions other than WG were mostly anti-PR 3 negative. The only patient with a high anti-PR 3 antibody value had classic periarteritis nodosa. The other six patients in this group had no systemic vasculitis: five of six were negative, one was borderline positive (21 AU, a patient with primary sclerosing cholangitis and sarcoidosis). The ppv of a positive anti-PR 3 result for WG is thus 11 of 13 or $85 \%$, for sys- 


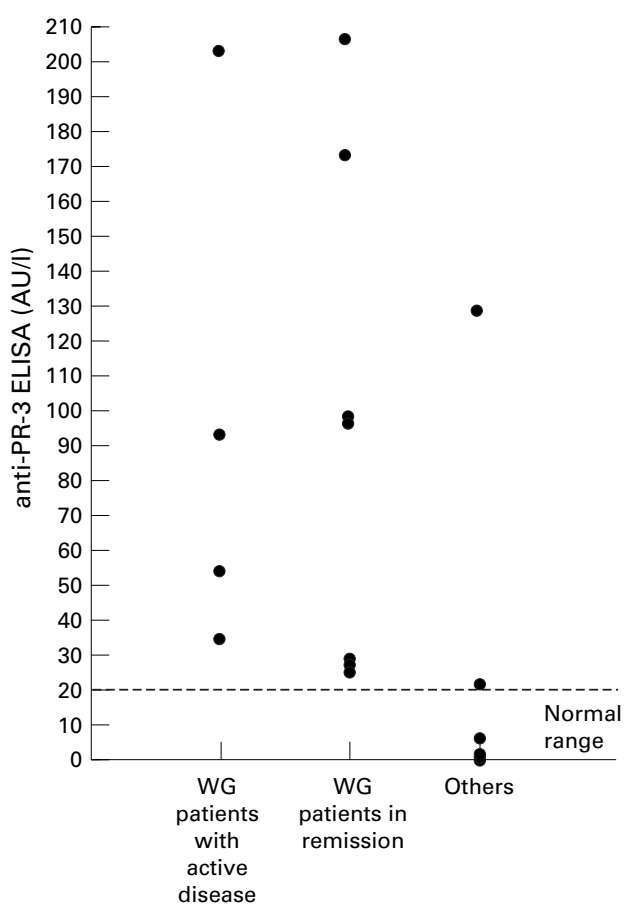

Figure 2 Highest anti-PR3 antibody values in four $W G$ patients with active disease, seven WG patients in remission, and in seven patients with conditions other than WG. The one high value in this last group was a classic periarteritis nodosa patient, the borderline positive value was in a patient with primary sclerosing cholangitis and sarcoidosis.

temic vasculitis $92 \%$ (including the patient with classic periarteritis nodosa).

Anti-MPO antibodies were measured in 10 patients with colitis ulcerosa (nine pure p-ANCAs and $1 \mathrm{c}$ - and p-ANCAs); they were negative in 9 of 10 . Anti-MPO antibodies were determined in all six vasculitis patients with pure p-ANCAs. In three of them, anti-MPO antibodies were present at a level higher than 5 AU/1. Anti-MPO antibodies were determined in nine patients with p-ANCAs without good explanation: they were negative in eight $(<5$ $\mathrm{AU} / \mathrm{l})$ and positive in one (11AU/1, a patient with combined c- and p-ANCAs) (table 1).

ANA AND IMMUNE COMPLEX DETERMINATION IN THE 94 PATIENTS

ANA determinations were available in 82 patients. ANA was present in 17 patients: $11 \%$ $(n=4)$ of pure c-ANCA serum samples were ANA positive (all homogenous patterns), $21 \%$ $(n=8)$ of pure $\mathrm{p}$-ANCA serum samples were ANA positive (also all homogenous pattern) while five serum samples (62\%) with combined c-and p-ANCAs were positive (three with a homogenous pattern, one nucleolar, one finely speckled pattern). Anti-DNA antibodies were negative in 12 of 13 ANA positive serum samples tested.

Serum immune complexes were determined in 38 patients, they were positive in $68 \%$ of these patients $(n=26): 74 \%$ of positive c-ANCA serum samples tested contained immune complexes, $55 \%$ of p-ANCA serum samples tested had immune complexes, and in $75 \%$ of serum samples with a combination of c- and p-ANCA antibodies, immune complexes could be demonstrated.

\section{Discussion}

Positive predictive value can be used to evaluate the usefulness of ANCA determination in clinical practice. ${ }^{9}$ However, preselection bias may considerably affect its value. ${ }^{10}$ Furthermore, the population studied should be defined unequivocally to save comparability with other studies. Therefore, we defined our study population as all ANCA positive patients who attended our department during one year. ANCA determinations were carried out only on clinical suspicion of ANCA related diseases.

Ninety four positive patients were found. In eight of them $(8.5 \%)$ both c- and p-ANCAs were present, which contrasts with previous statements that c- and p-ANCAs are mutually exclusive. ${ }^{11}$ It is remarkable that most of these patients $(62 \%)$ were also ANA positive.

We calculated a ppv of $40 \%$ for c-ANCAs in WG and of $56 \%$ in systemic vasculitis. This figure is independent of the antibody titre, while sensitivity of course dramatically drops when only considering higher c-ANCA titres as indicative for WG. This latter value should be compared with the ppv of $40 \%$ obtained by Davenport et $a l^{12}$ for a combination of WG, microscopic polyangiitis, and the syndrome of systemic vasculitis and with the $38 \%$ obtained by Edgar et $a l^{13}$ for any primary vasculitic disorder. In contrast with our experience, the ppv obtained by these authors increased by taking higher cut off points.

The ppv of a positive p-ANCA determination for a systemic vasculitic disorder was $18 \%$ (eight of 45 positive p-ANCA patients had systemic vasculitis), very similar to the $20 \%$ obtained by Edgar et al..$^{13}$ Almost half of our patients with positive p-ANCA determinations suffered from an inflammatory bowel disease, especially colitis ulcerosa, isolated or associated with primary sclerosing cholangitis (predictive positive value for these disorders $44 \%$ ).

In contrast with c-ANCA immunofluoresence results, a positive anti-PR 3 ELISA was very predictive of WG. Of 13 positive anti-PR 3 determinations, 11 were found in WG patients, one in a classic periarteritis nodosa patient and only one (borderline) positive determination in a non-vasculitic disorder (fig 2). This represents a ppv for WG of $85 \%$, for systemic vasculitis of $92 \%$. However, there was no correlation between the height of a positive ELISA test and the activity of the vasculitis in WG patients (fig 2)

During our study period, we had only a limited number of positive anti-MPO ELISA results: four of seven determinations were positive in p-ANCA positive vasculitis patients (including one WG patient with combined cand p-ANCA), one of 10 patients with inflammatory bowel disease and in one patient with bronchiectatic lung disease (with high c- and p-ANCAs). MPO is considered the main antigen responsible for p-ANCA staining in vasculitis patients, but other antigens may also be involved, for example, elastase, lactoferrin, etc. 
P-ANCAs in inflammatory bowel disease are generally directed against antigens other than $\mathrm{MPO}^{14}$

For three WG patients with active disease and seven WG patients in remission, ANCA determinations were carried out several times during the study period. We found substantial variations in titres with time. These were not correlated to disease activity, as these patients were in constant remission. Some patients in remission always had higher ANCA titres than other patients with still active disease. In a recently finished study in 50 Wegener patients, followed up in our department, ${ }^{15}$ we also could not find a correlation between ANCA titres and disease activity. Therefore, we do not treat patients based on variations in their ANCA titres, but rely on clinical and well documented biochemical parameters of disease activity. Kerr et $a l^{16}$ and Davenport et $a l^{17}$ were also unable to find a close relation between changes in ANCA titres and disease activity. ANCA assays should always be used in conjunction with other parameters of disease activity and should not be the sole basis for changing treatment. ${ }^{18}$

In 22 patients, we could not find an obvious cause for positive c-ANCAs. However, our attention was drawn to the fact that many of the different diseases found in these patients were chronic diseases that are frequently associated with polyclonal hypergammaglobulinaemia (for example, bronchiectasias, cirrhosis, Sjögren's syndrome, etc). Hyperglobulinaemia was present in 11 of 16 patients. We found a significant correlation between the gammaglobulinaemia and the c-ANCA titre in these 16 patients. All except one were anti-PR 3 negative. It is possible that part of our c-ANCAs are what is called "atypical ANCAs". Positive ANCA staining with a more homogenous rather than a granular cytoplasmic staining pattern was seen, for example, in patients with HIV. ${ }^{19}$ These atypical patterns are associated with antibodies to other antigens such as lactoferrin and elastase. ELISAs with these antigens are not available in our laboratory. In immunofluorescence tests, a clear distinction between these atypical ANCAs and true c-ANCAs is not always possible and relies highly on interpretation by the technician (granular versus more homogenous cytoplasmic fluorescence). In our laboratory and in many others, fine or more granular diffuse cytoplasmic staining patterns are all reported as c-ANCAs.

Hypergammaglobulinaemia, perhaps in combination with immune complex formation (found in most our patients), as a cause of false positive c-ANCA determinations may explain why patients with HIV or with Sjögren's disease, two disorders frequently associated with polyclonal hypergammaglobulinaemia, are sometimes ANCA positive. It may be a hypothesised that polyclonal B cell proliferation may result in the presence of these anti-PR 3 negative c-ANCAs (or atypical ANCAs), as was found in patients from the tropics with malaria or tuberculosis. ${ }^{20}$ We are however aware of the potential pitfalls of any retrospective approach. For instance, diagnosis was made in the knowledge of serological results and this might potentially have influenced the ppv obtained for ANCAs. Therefore, the results obtained in this study should be confirmed (or denied) by ANCA determinations in patients with hypergammaglobulinaemia and in different types of vasculitis.

1 van der Woude FJ, Rasmussen N, Lobatto S, et al. Autoantibodies against neutrophils and monocytes : tool for diagnosis and marker of disease activity in Wegener's granulomatosis. Lancet 1985;i:425-9.

2 Davies DJ, Moran JE, Niall JF, Ryan GB. Segmental necrotising glomerulonephritis with antineutrophil antibody : possible arbovirus aetiology? BMJ 1982;285:606.

3 Jenette JC, Falk RJ. Antineutrophil cytoplasmic autoantibodies and associated diseases : a review. Am J Kidney Dis 1990;15:517-29.

4 Niles JL, McCluskey RT, Ahmad MF, Arnaout MA. Wegener's granulomatosis autoantigen is a novel proteinase. Blood 1989;74:1888-93.

5 Falk RJ, Jennette JC. Anti-neutrophil cytoplasmic autoantibodies with specificity for myeloperoxidase in patients with systemic vasculitis and idiopathic necrotizing and crescentic glomerulonephritis. N Engl J Med 1988;318: 1651-7.

6 Rump JA, Scholmerich J, Gross V, et al. A new type of perinuclear antineutrophil cytoplasmic antibody (p-ANCA) in active ulcerative colitis but not in Crohn's disease. Immunobiology 1990;181:406-13.

7 Peter HH, Metzger D, Rump A, Röther E. ANCA in diseases other than systemic vasculitis. Clin Exp Immunol 1993;91 (suppl 1):S12-14

8 Leavitt RY, Fauci AS, Bloch DA, et al. The American College of Rheumatology 1990 criteria for the classification of lege of Rheumatology 1990 criteria for the classification of
Wegener's granulomatosis. Arthritis Rheum 1990;33: Wegener'

9 Edgar JDM. The clinical utility of ANCA positivity. Ann Rheum Dis 1996;55:494-6.

10 Jennette JC. Anti-neutrophil cytoplasmic autoantibodyassociated disease: a pathologist's perspective. Am J Kidney Disease 1991;18:164-70.

11 Geffriaud-Ricouard L, Noel LH, Chauveau D, Houhou S, Grünfeld JP, Lesafre P. Clinical spectrum associated with ANCA of defined antigen specificities in 98 selected patients. Clin Nephrol 1993;39:125-36.

12 Davenport A, Lock RJ, Wallington TB, Feest TG. Clinical significance of anti-neutrophil cytoplasm antibodies detected by a standardised indirect immunofluorescence assay. Q J Med 1994;87:291-9.

13 Edgar JDM, McMillan SA, Bruce I, Conlan SK. An audit of ANCA in routine clinical practice. Postgrad Med J ANCA in routine

14 Kallenberg CGM, Brouwer E, Weening JJ, Cohen Tervaert JW. Anti-neutrophil cytoplasmic antibodies: current diagnostic and pathophysiological potential. Kidney Int 1994; 46:1-15.

15 Blockmans D, Vandenbon C, Macken T, Stevens E, Bobbaers H. Wegener's granulomatosis : a study on 50 patients. European Journal of Internal Medicine 1997;8: 19-26.

16 Kerr GS, Fleisher TA, Hallahan CW, et al. Limited prognostic value of changes in anti-neutrophil cytoplasmic antibody titer in patients with Wegener's granulomatosis. Arthritis Rheum 1993;36:365-71.

17 Davenport A, Lock RJ, Wallington T. Clinical significance of the serial measurement of autoantibodies to neutrophil cytoplasm using a standard indirect immunofluorescence test. Am J Nephrol 1995;15:201-7.

18 De'Oliveira J, Gaskin G, Dash A, Rees AJ, Pusey CD. RelaDe'Oliveira J, Gaskin G, Dash A, Rees AJ, Pusey CD. Rela-
tionship between disease activity and anti-neutrophil cytoplasmic antibody concentration in long-term managemen of systemic vasculitis. Am J Kidney Dis 1995;25:380-9.

19 Klaassen RJL, Goldschmeding R, Dolman KM, et al. Antineutrophil cytoplasmic autoantibodies in patients with symptomatic HIV infection. Clin Exp Immunol 1992;87: 24-30.

20 Adebajo AO, Charles P, Maini RN, Hazleman BL. Autoantibodies in malaria, tuberculosis and hepatitis B in a West African population. Clin Exp Immunol 1993;92:73-6. 